\title{
A Computer Program for the 2-D Magnetostatic Problem Based On Integral Equations for the Field of the Conductors and Boundary $10 \mathrm{Sir}$
}

\author{
Gerry H. Morgan \\ Brookhaven National Laboratory, Upton, New York 11973
}

OCT 031991

\begin{abstract}
The iterative design of the 2-dimensional cross section of a beam transport magnet having infinitely permeable iron boundaries requires a last means of computing the field of the conductors. Solutions in the form of series expansions are used for rectangular iron boundaries [1], and programs based on the method of images are used to simulate circular iron boundaries. A single procedure or program for dealing with an arbitrary iron boundary would be useful. The present program has been tested with rectangular and circular iron boundaries and provision has been made for the use of other curves. It uses complex contour integral equations for the field of the constantcurrent density conductors and complex line integrals for the field of the piecewise-linear boundary elements.
\end{abstract}

\section{INTRODUCTION}

The present work was begun to fill the need for a small, fast program having the capability of finding the field of a 2-D current array in a closed region having arbitrary field-parallel or field-perpendicular boundaries. This is an old problem, for which many methods of solution have been developed. A number of analytical methods and some older numerical methods are given in [2] and some more recent numerical methods, most of which deal with the larger problem of non-linear iron, in [3]. The present program uses an integral-equation formulation to avoid meshing the conductors, and uses boundary elements just outside the problem domain to satisfy the boundary conditions. It was intended to be used to find field penetration profiles in superconductors obeying the critical-state model, with constant current density. The program has not been used for this, but may be useful in the early stages of magnet design.

\section{METHOD}

The methodology used is similar to that of the Rutherford program "GFUN2D" [4]. The field due to the sources and unknown boundary elements is required to satisfy the boundary conditions at every point, leading to a set of linear equations, the matrix of which is saturated.

Symmetry or asymmetry is dealt with by appropriate

Work performed under the auspices of the L'S. Department of Energy. funded in pan by the OESD - STEPS Program. choice of boundary type, field parallel $\left(\Gamma_{2}\right)$ or field perpendicular $\left(\Gamma_{1}\right)$, boundary position and conductor configuration. The two dipole examples presented below are one quadrant of the magnet. Another example might be one octant of a quadrupole inside circular iron; this would have a $\Gamma_{1}$ midplane boundary and a $\Gamma_{1}$ circle from it up to a forty-five degree $\Gamma_{2}$ line. A magnet consisting of an iron aperture with conductors inside having no symmetry would have a single $\Gamma_{1}$ boundary, which might be polygonal, circular or a mixture of lines and circular arcs. A circular dipole magnet baving no midplane symmetry but with left-right symmetry would have a $\Gamma_{2}$ vertical line on the left and a $\Gamma_{1}$ semicircle on the right; with left-right antisymmetry, the vertical line would be a $\Gamma_{1}$.

\section{A. The Boundary}

The boundary may consist of either straight lines or circular arcs or a combination. The input information required for a straight line is the beginning and end points and the desired number of segments into which it is to be subdivided. The information required for a circular arc is the beginning and end points, the number of segments and a third point which may be a point on the arc, the center of the arc, or the radius. Provision has been made for noncircular curves, but this is not yet implemented.

Each straight line or arc must be designated as either field-normal or field parallel. The $\Gamma_{1}$ boundary condition is that the imaginary part of $\hat{s}_{i} \cdot H_{1}$ be zero, where $\hat{s}_{i}$ is the unit tangent vector at the $\mathrm{i}^{\mathrm{th}}$ point, $\mathrm{H}_{\mathrm{i}}=\Sigma \mathrm{H}_{\mathrm{e}}+\Sigma \mathrm{H}_{\mathrm{s}}$ and $\Sigma \mathrm{H}_{e}$ and $\Sigma \mathrm{H}_{3}$ are the sums of the fields due to all the elements or sources, resp. Similarly, the $\Gamma_{2}$ boundary condition is that the real part of $\hat{s}_{i} \cdot H_{1}$ be zero. The unit tangent vector $\dot{s}_{1}=$ $\left(z_{1+1}-z_{1.1}\right) /\left|\left(z_{1+1}-z_{1-1}\right)\right|$.

\section{B. Elements}

Piecewise linear current and current dipole boundary elements were tried. The complex field $\mathrm{H}=\mathrm{H}_{\mathrm{y}}+\mathrm{i} \mathrm{H}_{\mathrm{x}}$ at $z_{0}$ due to a linear current element with density $\lambda=\lambda_{1}+\left(\lambda_{1+1^{-}}\right.$ $\left.\lambda_{1}\right) u$, where $u$ is a scalar parameter which is zero at $z_{1}$ and 1 at $z_{n+1}$, is given by $H=(1 / 2 \pi) \int\left[\lambda /\left(z_{n}-z\right)\right] d s$, where ds $=$ $\left|\Delta z_{1}\right|$ du and $\Delta z_{1}=z_{1} \cdot 1-z_{1}$ (MKS units). Carrying out the integration over $\mathrm{u}=0$ to 1 vields $(1)$.

Similarly, the field due to a linear dipole element is given by $H=(i / 2 \pi) \int\left[m \hat{e} /\left(z_{i j}-z\right)^{2}\right] d: i$, where $m$ is the scalar 
dipole density with direction given by the unit vector

$$
\begin{aligned}
& H-\frac{\lambda_{i}}{2 \pi}\left[\log \left(\frac{z_{0}-z_{i}}{z_{0}-z_{i+1}}\right)+\eta\right] \frac{|\Delta z|}{\Delta z}-\frac{\lambda_{i+1}}{2 \pi} \eta \frac{|\Delta z|}{\Delta z} \\
& \text { where } \eta-1-\frac{z_{0}-z_{i}}{z_{i+1}-z_{i}} \log \left(\frac{z_{0}-z_{i}}{z_{0}-z_{i+1}}\right)
\end{aligned}
$$

$\hat{\mathrm{e}}=\hat{a} \Delta \mathrm{z}_{\mathrm{i}} /\left|\Delta \mathrm{z}_{\mathrm{i}}\right|$. With $\mathbf{m}=\mathbf{m}_{\mathrm{i}}+\left(\mathbf{m}_{\mathrm{i}+1}-\mathbf{m}_{\mathrm{i}}\right) \mathbf{u}$ and $\mathbf{a}$ constant, carrying out the integration gives (2).

$$
\begin{gathered}
H-\frac{i a}{2 \pi\left(z_{i+1}-z_{i}\right)}\left\{m_{i}\left[-\frac{z_{i+1}-z_{i}}{z_{0}-z_{i}}+\log \left(\frac{z_{0}-z_{i}}{z_{0}-z_{i+1}}\right)\right]\right. \\
\left.-m_{i+1}\left[-\frac{z_{i+1}-z_{i}}{z_{0}-z_{i+1}}+\log \left(\frac{z_{0}-z_{i}}{z_{0}-z_{i+1}}\right)\right]\right\}
\end{gathered}
$$

Whichever type is used, the elements are positioned just outside the boundary, on lines parallel to it; all lines are spaced the same distance from the boundary equal to a factor $f_{d}$ times the length of the smallest boundary segment. These element lines constitute a closed path around the boundary. The segmentation of the element lines is similar to that of the boundary lines.

Either element type may be used exclusively or both may be used in the same problem. If both are used, the point marking the junction between segments of two types must consist of two superimposed points, otherwise there are two unknown densities associated with the same point.

In the latter stages of program development, it was found that the solution was sensitive to the spacing between ihe boundary and the elements; changing $f_{d}$ in it's least significant digit caused a large change in the solution, i.e., a chaotic behavior. This was attributed to the near-coincidence of some of the boundary and element points and the $1 / \mathrm{r}$ behavior of (1) near an element end point. The problem was essentially eliminated by varying the element segment length randomly, requiring only that the line end points remain fixed. A slightly different solution is obtained each time the random number generator is reset.

With randomization of element segment size, $f_{d}$ was varied from 1 to 4 , noting for each value the quality of the solution on the boundary, especially the deviations from the known solution and the tendency towards divergence near corners. Solutions were better for $f_{d}$ between 1.5 and 3, particularly just above 2 , so $f_{d}=2.1$ was adopted for both exampies described below.

\section{Sourc:}

The field due to the sources is computed using the expression obtained by Beth [5], given in (3), for the field at $\mathrm{z}_{\mathrm{j}}$ of a polygonal region of $\mathrm{n}$ sides with vertices $\mathrm{z}_{1}$ enclosing an area having constant-current density $\sigma$. This expression is implemented in subroutine ALFLD.

$$
\begin{gathered}
H\left(z_{0}\right)-\frac{i \sigma}{4 \pi} \sum_{i-1}^{n} \alpha_{i} \log \left(\frac{z_{i}-z_{0}}{z_{i-1}-z_{0}}\right), \\
\text { where } \alpha_{i}-\left(z_{i}-z_{0}\right)^{\circ}-\left(z_{i}-z_{0}\right)\left(\frac{\Delta z_{i-1}}{\Delta z_{i-1}}\right)
\end{gathered}
$$

\section{The Set of Linear Equations}

The unknown quantities are the $\mathrm{N}_{\mathrm{e}}$ current densities $\lambda_{1}$ at the ends of the element segments. The $i^{\text {th }}$ boundary point is assumed to lie at the beginning of the $\mathrm{i}^{\mathrm{it}}$ segment. A corner point has associated with it two boundary conditions, except in the case of $\Gamma_{1} \Gamma_{3}$ corners for which the included angle is $\pi / 2$ or $3 \pi / 2$. With this exception, all boundary corner points are doubled, i.e., a second point is coincident with the original, so that a line ending at a corner has a point at the end of the final segment. For each such extra point, an element point and element segment are added to the corresponding element line, in order to keep the number of equations equal to the number of unknowns. The present version uses only current elements; if both current and dipole elements were present, requiring doubling of element points at the junction of unlike lines, an extra boundary segment and point would have to be added for each junction.

In configurations having two separate $\Gamma_{2}$ sides, the change in scalar potential $\left(\int \mathrm{H} \cdot \mathrm{ds}\right)$ in each such side, when summed, equals the total current $I$ in the sources. (A side may consist of several lines.) The scalar potential is apportioned between the two $\Gamma_{2}$ sides by an additional constraint equation: $\int \mathrm{H} \cdot \mathrm{ds}=f \mathrm{I}$, where the integral is taken along one of the sides and $f$ is between zero and one. In this case, an additional element point and segment is added to keep the matrix square. The indicated integral is done using Simpson's rule coefficients in the extra equation.

An additional complication occurs in the setting up of the matrix: there are two additions to the coefficient of each unknown because both $\lambda_{1}$ and $\lambda_{1+1}$ appear in (1). A typical matrix element for a $\Gamma_{1}$ boundary point is $M_{i j}=\operatorname{imag}\left(G_{1, j}\right)$ $+\operatorname{imag}\left(G_{2,-1}\right)$, where $G_{1 \jmath}=\hat{s}_{j}$ times the coefficient of $\lambda_{i}$ in (1) and $G_{2 j-1}=\hat{s}_{j-1}$ times the coefficient of $\lambda_{1+1}$ in (1). A matrix element for a $\Gamma_{2}$ boundary uses the negative of the real part of $\mathrm{G}$.

The set of linear equations may be expressed as $\mathbf{M} \mathbf{x}=$ $y$, where $y_{1}$ is the real, or minus the imaginary, part of $\left(\hat{\mathrm{s}}_{1} \cdot \Sigma \mathrm{H}_{\mathrm{s}}\right)$. The set is solved using $L U$ decomposition of $\mathbf{M}$ followed by forward and back substitution, with iterative improvement; published routines [6] LUDCMP,LUBKSB and MPROVE are used for this.

\section{RESULTS}

Two examples are given; the first has a rectangular iron aperture with current to the right, touching the iron on the top, right and bottom, a "picture-frame magnet", Figure 1. This example has a simple analytic solution. 


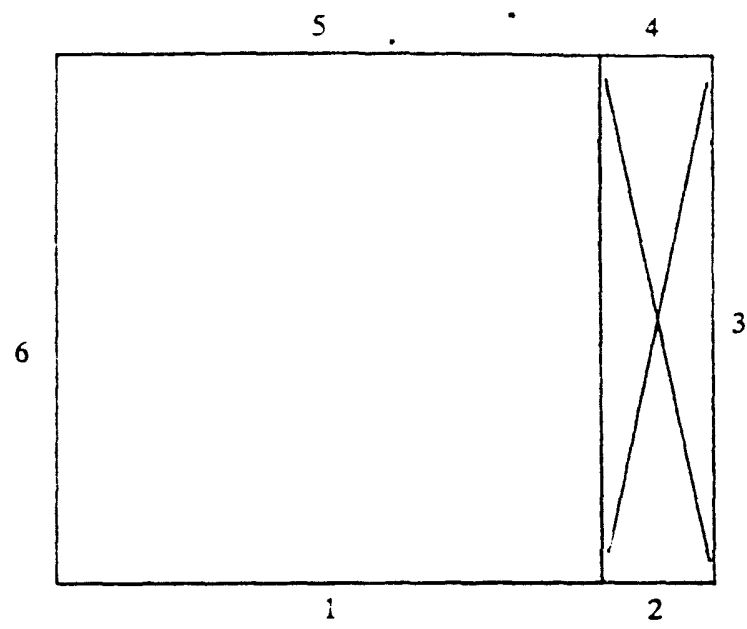

The Picture Frame Dipole

Figure 1

The second has a round iron aperture with a small rectangular current located at a radius less than that of the iron, and 30 degrees up from the midplane, the 2-D analog to the circular "Helmholtz coil", Figure 2. This second example has an exact numerical solution using an algorithm similar to that of (3), but with an added term for the image of the current in a circular, $\Gamma_{1}$ boundary [7].

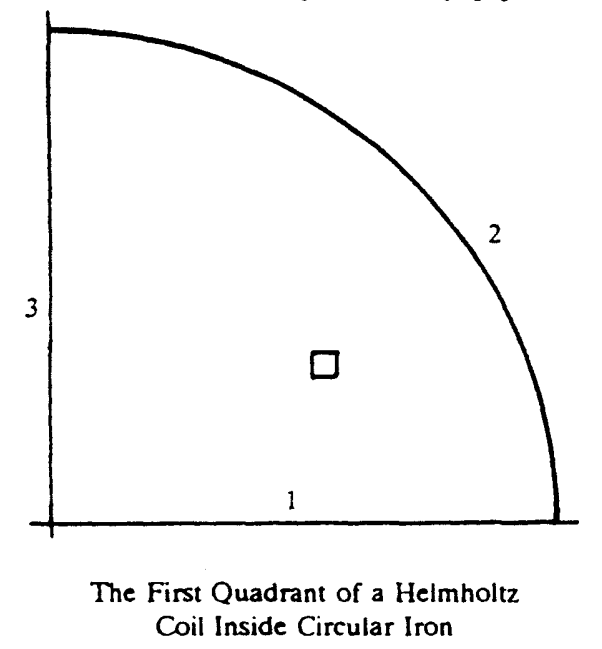

Figure 2

All the program development was done using the first example, a fortunate choice because the corners, the current touching the boundary, and the two $\Gamma_{2}$ sides (left and right) are challenging to this method of solution. The quality of a solution is assessed by examining the field at the boundary points.

\section{A. The Picture-Frame Dipole}

The first example, Figure 1, has basically 6 lines, conveniently numbered courterclockwise starting at the origin. Lines 3 and 6 are $\Gamma_{2}$; this simulates a periodic problem. Line 3 could equally well be $\Gamma_{1}$ to simulate an iron boundary. A plausible subdivision would be about equal on lines 1,3,5 and 6 , and somewhat finer on 2 and 4 , because the field magnitude is known to drop linearly to zero in the conductor in this basically one-dimensional problem. Aiming for a total segmentation of about 100 , one might try 20,10 , $20,10,20,20$. Although the solution is essentially correct, this segmentation results in substantial oscillation of the field near the corners at the ends of line 3.

A good result is obtained with 96 segments by decreasing the segment length in several segments near each corner, by about a factor of two compared to the rest of the line. A very good result is obtained by also decreasing the segment length on each side of the junction between the regions where current touches the boundary and where it does not. Each of the original 6 lines is subdivided into three lines, and the short lines at the beginning and end each contain 4 segments. The remaining parts of the original 6 lines contain 12, 4, 14, 4, 12 and 14 segments, resp. This results in 108 segments on the boundary. Figure 3 shows the magnitude of the difference between the analytic solution and the result from this latter case, at all points on the boundary, as a fraction of the magnitude of the field at the origin. As can be seen, the maximum error is about $5 \times 10^{-3}$. The rms error is $8.0 \times 10^{-4}$.

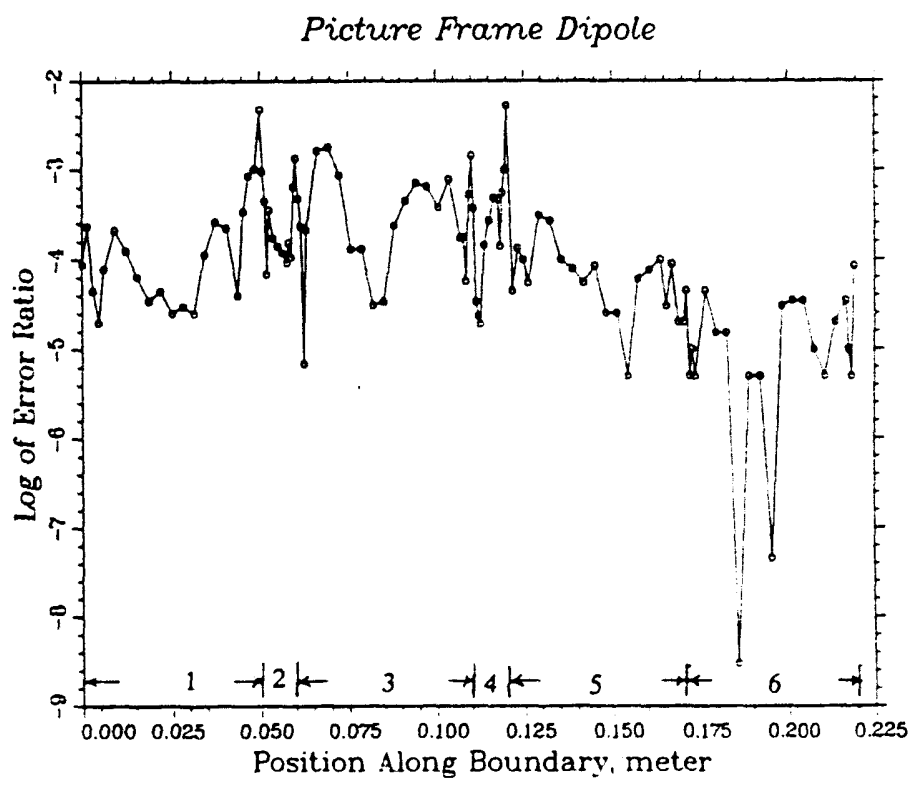

Figure 3

\section{B. Circular Iron Around a Helmholtz Dipole}

A basic segmentation of $24,32,24$ for the three lines comprising the boundary gives an acceptable solution, but with an $0.5 \%$ error at the upper left corner. Decreasing the segment length at the end of each line as was done for the picture frame magnet gives an excellent solution. The segmentation is then $4,18,4,4,28,4,4,18,4$, for a total of 88. Figure 4 shows the magnitude of the difference between 
the exact solution and the program output, as a fraction of the magnitude of the field at the origin. The maximum error is $6 \times 10^{-4}$ and the rms error is $1.0 \times 10^{4}$.

Circular Iron Around Helmholtz Dipole

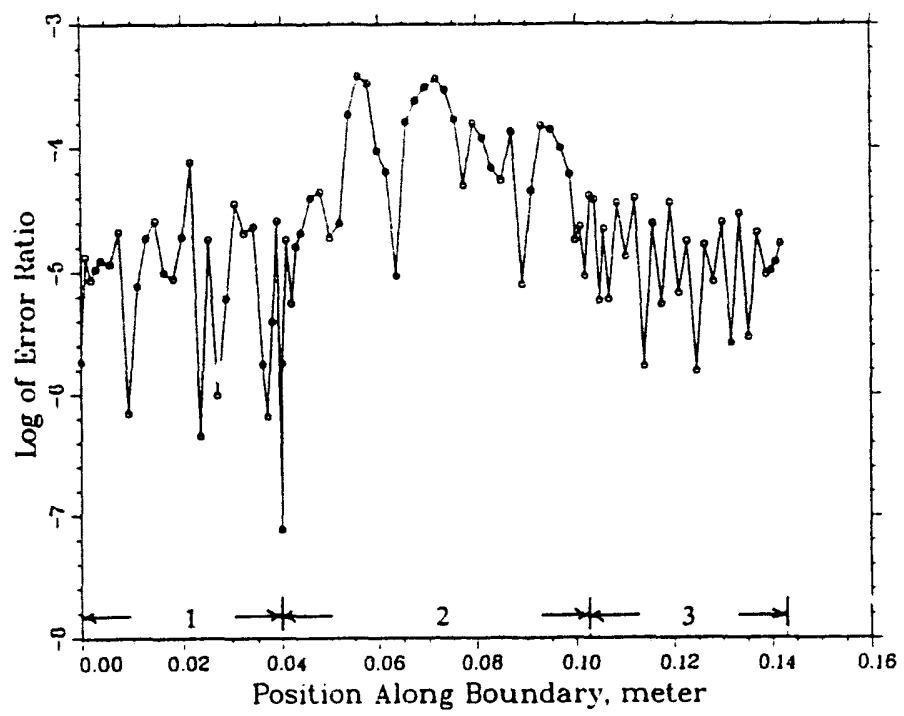

Figure 4

\section{Timing}

The computer used is a VAX 11/785, used single precision (about 7 decimal digits). The times required for the various steps in the 108 segment picture frame example are given in Table 1 . "H on bndry" is the output.

TABLE 1

TIME IN SECONDS FOR VARIOUS OPERATIONS

\begin{tabular}{||l|l|l|l|}
\hline Operation & Time & Operation & Time \\
\hline \hline Draw source & 0.07 & Draw bndry & 25.28 \\
\hline Hs $_{\text {s }}$ on bndry & 0.09 & setup matrix & 6.01 \\
\hline LUDCMP & 1.42 & LUBKSB & 0.06 \\
\hline MPROVE (2) & 0.18 & H on bndry & 4.10 \\
\hline
\end{tabular}

The time for drawing the sources and the boundaries goes as $N$, the number of segments. The time for setting up the matrix goes as $\mathrm{N}^{2}$. The time for the $L U$ decomposition in LUDCMP goes as $\mathrm{N}^{3}$. Once the $L U$ decomposition is complete, however, a new source configuration requires only the execution of LUBKSB, which is much quicker. The time for setting up the source vector, " $\mathrm{H}_{\mathrm{s}}$ on bndry", is proportional to the number of sources; in an iterative improvement of a source configuration consisting of say, 40 turns, this would amount to 3.6 seconds. Part of the 0.09 seconds shown for one source, however, is due to $1 / 0$ time.

It would be difficult to make a timing comparison with other available programs; these are a circular iron aperture program using the image method, which would be expected to be considerably faster that the present program, and a finite element program which can be used with an $\infty \mu$ boundary which would be expected to be much slower than the present program. It should be noted that iteratively varying a conductor configuration while using the present program would only require rerunning "draw source", LUBKSB and MPROVE, all of which are relatively fast.

\section{DISCUSSION}

The results show that the quality of the solution at the ioundary is dependent on the segmentation, for a roughly constant number of segments. No attempt has yet been made to automate the segmentation process, but the examples demonstrate how this could be done: subdivide each basic line into three, with normal spacing in the bulk of the segments near the middle, and four, half-length segments at the ends. Lines thought to require it could be assigned shorter segments, e.g., the places where the current touches the boundary on the top and bottom in the first example. A more complicated boundary having notches or protuberances might require custom segmentation in those are

For magnet design use, the multipole expansion of the field is desireable. This and a few other aids and output features, such as calculation of the field at specified points or lines and determination of the peak field will be added.

\section{REFERENCES}

[1] Richard J. Thome and John M. Tarrh "MHD and Fusion Magnets, Field and Force Design Concepts" John Wiley \& Sons, 1982

[2] K.J. Binns and P.J. Lawrenson, "Analysis and Computation of Electric and Magnetic Field Problems", Pergamon Press, $2^{\text {nd }}$ Ed. 1973

[3] M.V.K. Chari \& P.P. Silvester, ed.. "Finite Eiements in Electrical and Magnetic Field Problems", John Wiley \& Sons, 1980

[4] M.J. Newman. C.W. Trowbridge and L.R. Tumer, Proc. $f^{\text {th }}$ Int. Conf. on Magnet Technolog'. 1972

[5] R.A. Beth. J. Appl. Phys. Vol. 40, pp. 4782.1969

[6] W.H. Press, B.P. Fiannery, S.A. Teukolsky and W.T. Vetterling,

"Numerical Recipes". Cambridge Unvietsiny Press, 1986

[7] K. Halbach. Nucl. Instr. and Methods. Vol. 78, pp. 185. 1970

\section{DISCLAIMER} This report was prepared as an account of work sponsored by an agency of the United States
Government. Neither the United States Government nor any agency thereof, nor any of their
employees, makes any warranty, express or implied, or assumes any legal liability or responsi-
bility for the accuracy, completeness, or usefulness of any information, apparatus, product, or
process disclosed, or represents that its use would not infringe privately owned rights. Refer-
ence herein to any specific commercial product, process, or service by trade name, trademark,
manufacturer, or otherwise does not necessarily constitute or imply its endorsement, recom-
mendation, or favoring by the United States Government or any agency thereof. The views
and opinions of authors expressed herein do not necessarily state or reflect those of the
United States Government or any agency thereof. 

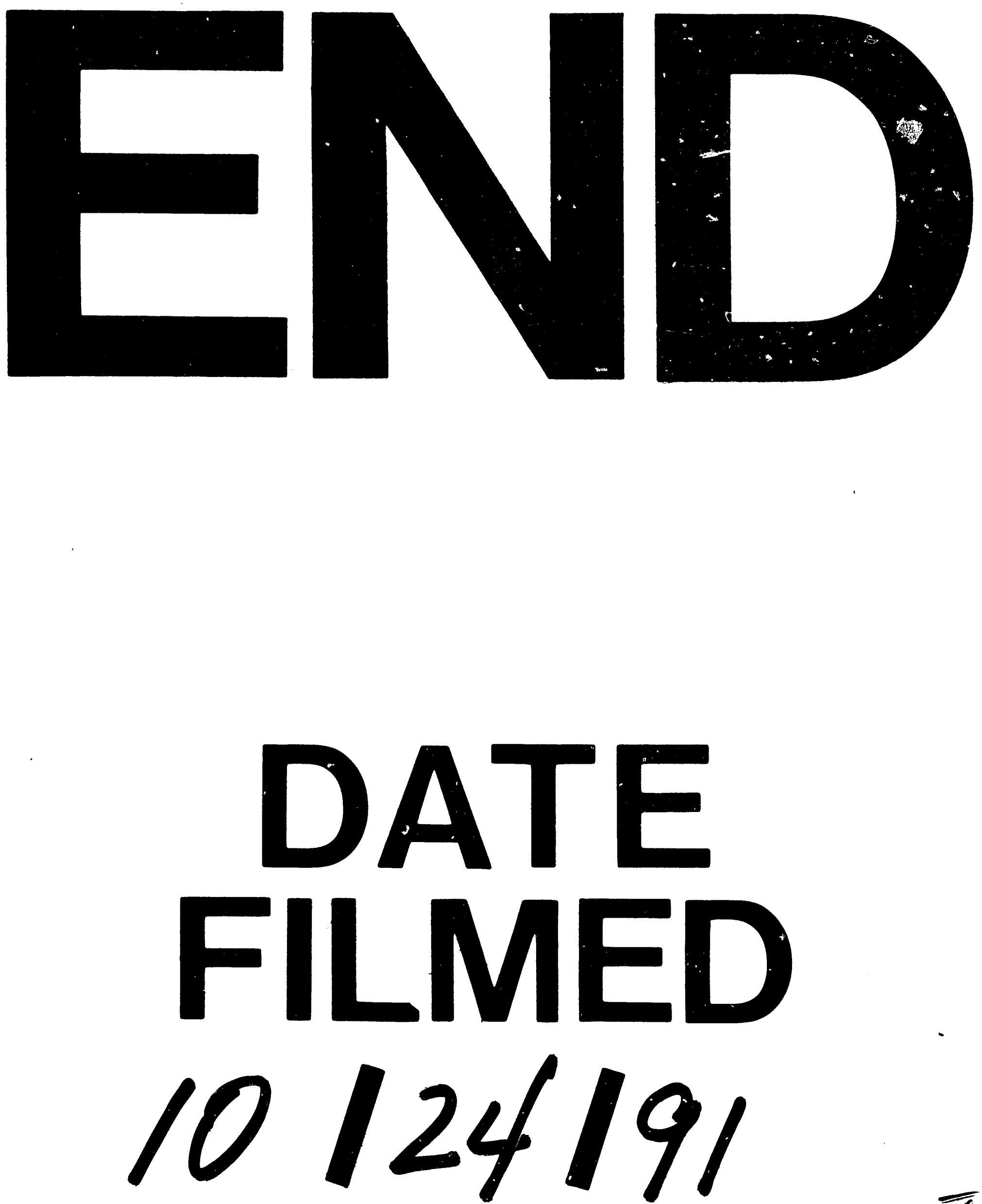

$\bar{I}$ 
$-$ 